\title{
Symptomatic cystic seminal vesicle: a laparoscopic approach for effective treatment
}

\author{
Anmar Nassir, MD, FRCSC
}

\begin{abstract}
This case report highlights a symptomatic cystic seminal vesicle lesion, treated laparoscopically. A young adult male known to have congenital right renal agenesis presented with a history of recurrent right iliac fossa pain, as well as deep pelvic discomfort. The preoperative evaluation revealed a $7 \times 4.5 \times 4 \mathrm{~cm}$ cystic seminal vesicle mass. After discovery of the mass, the patient underwent a transperitoneal laparoscopic excision of the lesion. The patient was discharged on the third postoperative day and had an uneventful recovery. There were no complications noted during a routine follow-up. The laparoscopic approach was shown to be effective in the management of this rare condition.
\end{abstract}

Can Urol Assoc J 2009;3(6):E81-E83

\section{Introduction}

Cysts of the seminal vesicle are uncommon. Although mostly asymptomatic, these cysts can present with varying degrees of different symptoms. ${ }^{1}$ This report highlights a case of a symptomatic right cystic seminal vesicle lesion associated with ipsilateral renal agenesis. In this case, the cyst was managed using a laparoscopic approach.

\section{Case report}

A single 24-year-old man known to have congenital right renal agenesis presented with acute abdominal pain to the emergency room. The patient was complaining of increasing pain at the right iliac fossa and deep pelvic discomfort associated with dysuria and frequency with two similar episodes in the past. Upon physical examination, he had low-grade fever and a tender palpable pelvic mass that was soft to firm in consistency on bimanual examination. In addition, both testes and the vas deferens felt normal. His blood workup revealed a leukocytosis level of 14000 . Ultrasound showed an irregular hypoechoic mass occupying the right side of the pelvis and absence of the ipsilateral kidney, but a normal left kidney. The mass did not show any evidence of vascularity by colour Doppler study (Fig. 1). Further evaluation using computed tomography scans showed that the mass was inseparable from the seminal vesicle area (Fig. 2). Magnetic resonance imaging confirmed these findings (Fig. 3). A mercapto acetyl triglycine (Mag)-3 radioactive nuclear renogram revealed that only the left kidney was functioning normally, with no uptake anywhere else in the body.

The patient was scheduled for elective transperitoneal laparoscopic excision after a course of antibiotic treatment.

The procedure was performed successfully using the standard pelvic laparoscopic ports placement along an inverted $\mathrm{V}$ line between the umbilicus and both anterior superior iliac spines. With an 8 to10 cm distance between ports, the following ports were inserted: a $10-\mathrm{mm}$ camera port near the umbilicus, a 10-12-mm port inferiolaterally to the right, and two 5-mm ports on the left side. With the surgical table placed in the Trendelenburg position, the bladder was retracted anteriorly and the cyst was easily identified by a transverse incision in the retrovesical peritoneum. The right vas deferens was identified and dissected medially to the ampulla and was used as a guide to the seminal vesicle. The dissection of the mass from the surrounding structure was made easier by maintaining good hemostasis with the help of a 5-mm bipolar LigaSure (Valleylab, Boulder, $\mathrm{CO})$. After careful dissection, the right vas deferens was not only inseparable, but was entering the cystic mass. It was clipped and divided. The whole lesion was excised at the level of the ipsilateral ejaculatory duct (Fig. 4) and retrieved by widening the 12-mm port enough to completely extract the specimen. The operation time was 130 minutes, with minimal blood loss. The patient was discharged on the third postoperative day, and had no complaints during a routine follow-up visit.

Pathological analysis of the pelvic mass revealed a multilocular cyst lined focally by benign-looking epithelium with focal papillary projection. The cells were cuboidal and columnar, with pale esinophilic to clear cytoplasm and brownish pigment. The wall of the cyst was composed of a well-formed smooth-muscle layer. There was no evidence 


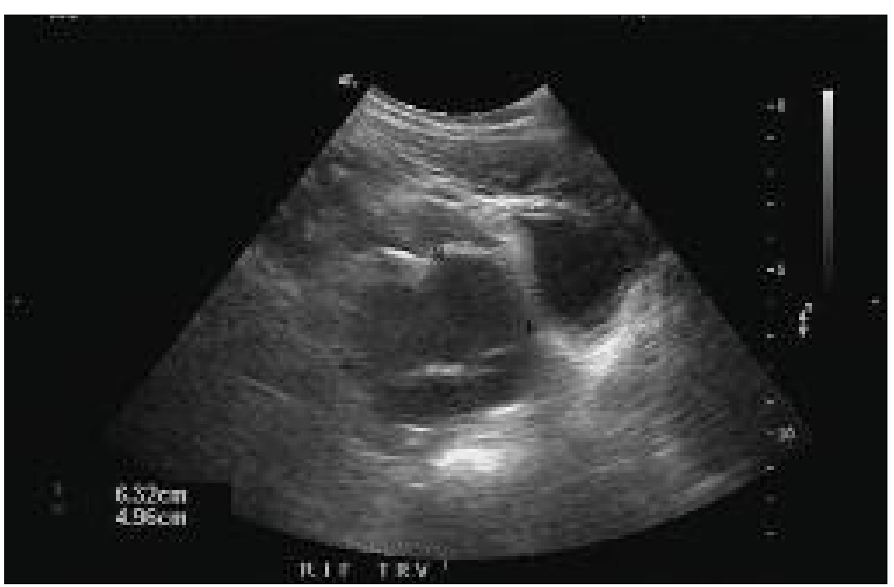

Fig. 1. Pelvic ultrasound showing a large cyst of the seminal vesicle measuring $6.3 \times 5 \times 6.7 \mathrm{~cm}$ with a central area of increased echogenicity.

of malignancy. Immunohistochemistry studies showed that the lining epithelium was positive for $\mathrm{CK} 7$ and focally for CK5 and CK6. It was negative for CK20, calretinin, vemintin and prostate-specific antigen.

\section{Discussion}

Congenital lesions of the seminal vesicle are rare, ranging from $0.002 \%$ in child health screening ${ }^{2}$ to $0.055 \%$ in an autopsy series of adolescent males. ${ }^{3}$ Congenital lesions are found in only $0.005 \%$ of the adult population. ${ }^{4}$ About 200 cases of seminal vesicle cysts have been reported to date. ${ }^{5}$

As a result of the interaction between the ureteral bud and the mesonephric duct during embryogenesis, many associated anomalies were observed. These anomalies are associated with ipsilateral renal agenesis in $70 \%$ of patients. ${ }^{1,6}$ Other anomalies may include renal dysplasia, ectopic ureteral orifice, hydroureter or vertebral deformity. Congenital seminal vesicle cysts are typically asymptomatic and rarely change during long-term follow-up examinations. ${ }^{6}$

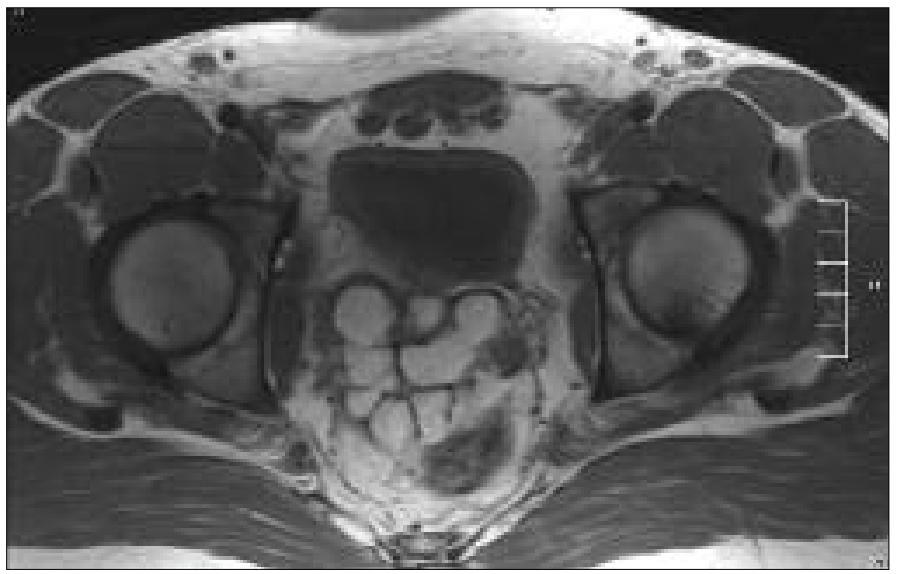

Fig. 3. Magnetic resonance imaging of the pelvis, showing a cystic dilatation of the right seminal vesicle. The urinary bladder and other surrounding structures appeared normal.

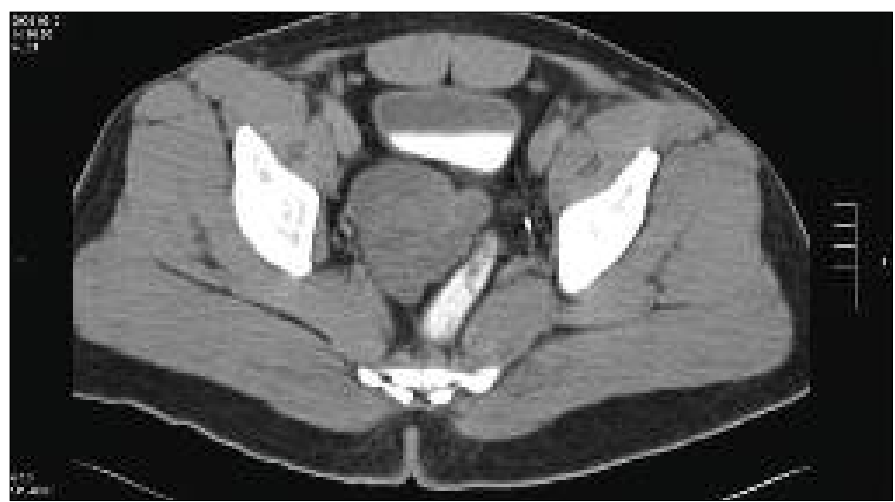

Fig. 2. Computed tomography scan of the pelvis revealing a $6.5 \times 6.7 \mathrm{~cm}$ lobulated soft tissue mass with faint internal septation and no calcification occupying mainly the right side of the pelvis between the recto sigmoid and urinary bladder. It showed minimal enhancement after intravenous contrast injection.

When symptomatic, these cysts usually present between the second and fourth decades of life. The symptoms are perineal in origin and predominantly consist of pain. Storage and voiding symptoms may also occur. Constipation, recurrent urinary tract infections, epididymitis and chronic prostatitis have also been reported as possible symptoms. In addition, other patients may present with infertility or sexual dysfunction. Very large masses may be palpable abdominally, while many smaller cysts are palpable rectally. Infected cysts may also present with symptoms of sepsis in addition to the symptoms described above. ${ }^{7-9}$

A review of the literature identified 79 males who underwent surgery for this entity. ${ }^{8}$ Several treatment options have been described, ranging from endoscopic aspiration to open vesiculectomy. Surgical excision of the seminal vesicle cyst is the preferred definitive management because simple aspiration is often complicated by a recurrence of the cyst and

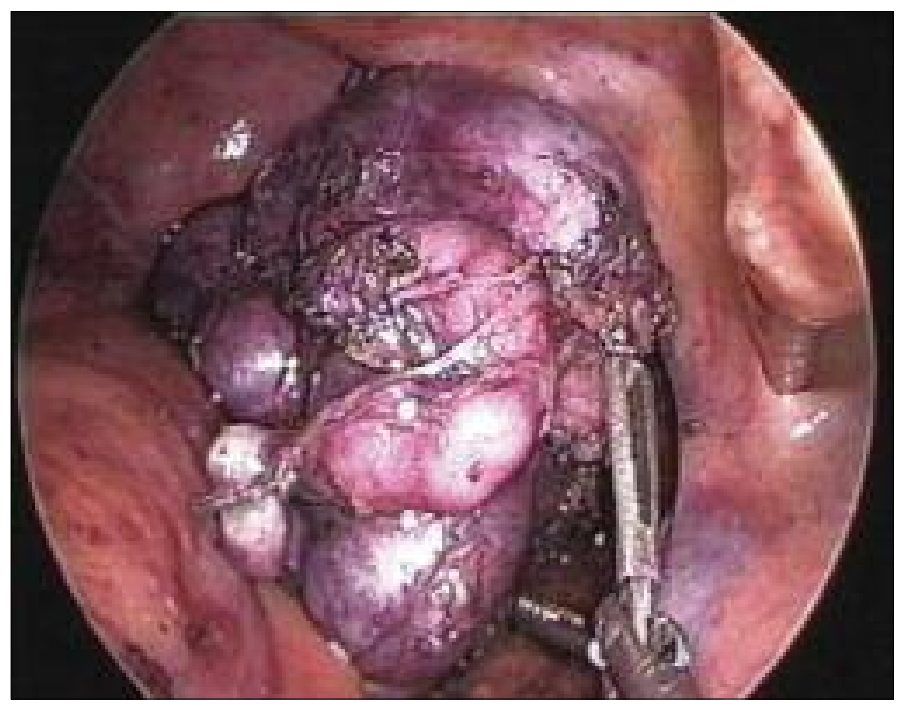

Fig. 4. Laparoscopic view of the cystic mass of the seminal vesicle dissected from the surrounding structure. The vas is seen clipped and folded on itself at the bottom of the image. 
infection of the area. ${ }^{10}$ To date, about 11 cases have been operated on laparoscopically. ${ }^{9}$ The technique we described, with little modification, was mentioned in the current literature. ${ }^{8,11,12}$ The bipolar ligature made our dissection easier and resulted in less oozing and better progression.

This approach is ideally suited for this procedure, with decreased postoperative pain and convalescence, combined with excellent exposure of the pelvic structures. In this case, the surgery took approximately 2 hours and the patient spent a total of 3 days in the hospital. His postoperative course was uneventful; his symptoms were resolved, with no recurrence of his initial symptoms after 18 months. These results are comparable to the overall average parameters reported by others: an operative time of 2.9 hours, an average hospital stay of 2.2 days and complete resolution of all symptoms at an average follow-up of 17 months. 8,9

Some authors have claimed that a robotic technique has the advantages of a higher degree of freedom with reproducibility and a shortened learning curve. ${ }^{4}$ Bearing in mind the expensive disposables and the added running cost of the system, the use of the robot is not convincing for similar ablative procedure lacking any reconstructive component.

\section{Conclusion}

Transperitoneal laparoscopic excision of seminal vesicle cysts should be considered as a treatment of choice for the management of symptomatic seminal vesicle cysts, even if they are sizable, because of its minimal invasiveness, short postoperative course and complete early recovery.

Assistant Professor of Urology, Um Al-Qura University, King Faisal Specialist Hospital \& Research Center, Kingdom of Saudi Arabia
Competing interests: None declared.

This paper has been peer-reviewed.

\section{References}

1. Altunrende F, Kim ED, Klein FA, et al. Seminal vesicle cyst presenting as rectal obstruction. Urology 2004;63:584-5.

2. Sheih $C P$, Hung $C S$, Wei $C F$, et al. Cystic dilatations within the pelvis in patients with ipsilateral renal agenesis or dysplasia. J Urol 1990;144:324-7.

3. Van den Ouden D, Blom JH, Bangma C, et al. Diagnosis and management of seminal vesicle cysts associated with ipsilateral renal agenesis: a pooled analysis of 52 cases. Eur Urol 1998;33:433-40.

4. Carmack AJ, Siddiq FM, Leveillee RJ. Novel use of da Vinci Robotic Surgical System: removal of seminal vesicle cyst in previously dissected pelvis. Urology 2006;67:199.

5. Manousakas T, Kyriakou G, Serafetinides E, et al. Partial vesiculectomy in an infertile man with seminal vesicle cyst, ipsilateral renal agenesis, and cryptorchidism. Urology 2002;59:602.

6. Chen HW, Huang SC, Li YW, et al. Magnetic resonance imaging of seminal vesicle cyst associated with ipsilateral urinary anomalies. J Formos Med Assoc 2006; 105:125-31.

7. Patel B, Gujral S, Jefferson K, et al. Seminal vesicle cysts and associated anomalies. BJU Int 2002;90: 265-71.

8. Cherullo EE, Meraney AM, Bernstein LH, et al. Laparoscopic management of congenital seminal vesicle cysts associated with ipsilateral renal agenesis. J Urol 2002;167:1263-7.

9. Moudouni SM, Tligui M, Doublet JD, et al. Laparoscopic excision of seminal vesicle cyst revealed by obstruction urinary symptoms. Int I Urol 2006;13:311-4.

10. Adeyoju AB, Taylor P, Payne SR: Congenital seminal vesicle cysts: an unusual but treatable cause of lower urinary tract/genital symptoms. BJU Int 2001;87:901-2.

11. Liatsikos EN, Lee B, Filos KS, et al. Congenital seminal vesicle cyst and coexisting renal agenesis: laparoscopic approach. Urology 2004;63:584-6.

12. McDougall EM, Afane JS, Dunn MD, et al. Laparoscopic management of retrovesical cystic disease: Washington University experience and review of the literature. J Endourol 2001;15:815-9.

Correspondence: Dr. Anmar Nassir, P0 Box 126143, Jeddah, 21352, Saudi Arabia; fax: 966-26744321; DrAnmar@gmail.com 